\title{
De cortesanos y burgueses. Los nacionalismos como motor de la conservación y restauración
}

\author{
$\mathbf{M}^{\mathrm{a}}$ Teresa Vicente Rabanaque, Beatriz Santamarina Campos, Virginia Santamarina Campos
}

\begin{abstract}
Resumen: Este artículo analiza el origen y desarrollo de los restauradores decimonónicos en el ámbito institucional español, a partir del estudio de caso de las ciudades de Madrid y Barcelona, como principales exponentes del desarrollo de la restauración española. Por un lado, la constitución de Madrid como capital política condicionó su mayor relevancia cultural, siendo pionera en la configuración del perfil profesional. Además, el centralismo madrileño justificó el protagonismo de la Real Academia de San Fernando como principal órgano de control de las restauraciones y del patrimonio. Por otro lado, Barcelona se erigió en el epicentro de la primera Revolución Industrial en España, hecho que repercutió en la incorporación de nuevos avances científicos en la disciplina de la restauración. Además, el desarrollo del nacionalismo catalán, como respuesta al centralismo castellano, le revistió de unas particularidades propias. En este texto se presentan los rasgos en la gestación de dos modelos diferenciados del restaurador institucional.
\end{abstract}

Palabras clave: Restauradores, Madrid, Barcelona, institucionalización, centralismo, nacionalismo, especialización profesional.

\section{De cortesãos e burgueses. Os nacionalismos como motor da conservação e restauro.}

Resumo: Este artigo analisa a origem e o desenvolvimento dos restauradores no século XIX, no âmbito institucional espanhol. A partir do estudo das cidades de Madrid e Barcelona como expoentes principais do desenvolvimento do restauro em Espanha. Por um lado, a constituição de Madrid como capital política proporcionou uma maior relevância cultural, sendo pioneira na configuração do perfil profissional. Para além disso, o centralismo de Madrid justificou o protagonismo da Real Academia de San Fernando como orgão principal de controlo das intervenções de restauro e do património. Por outro lado, Barcelona estava no epicentro da primeira Revolução Industrial em Espanha, facto que se repercutiu na adopção de novos avanços científicos no campo do restauro. O crescimento do nacionalismo catalão, como resposta ao centralismo castelhano, também o revestiu de certas particularidades próprias. Neste texto apresentam-se as características de nascimento de dois modelos diferenciados de restaurador institucional.

Palavras-chave: restauradores, Madrid, Barcelona, institucionalização, centralismo, nacionalismo, especialização profissional.

From nobles and bourgeois. Nationalisms as engine of conservation and restoration

Abstract: This articles analyses the origin and development of nineteenth century restorers in the scope of Spanish institutions, based on the case study of the cities of Madrid and Barcelona, as the main exponents of the deployment of Spanish restoration. On the one hand the constitution of Madrid as the political capital would condition its major cultural relevance, as a pioneer in the configuration of the professional profile. Furthermore, the centralism of Madrid would justify protagonism by the Royal Academy of St. Fernando as the main controlling body of restorations and heritage. On the other hand, Barcelona would become the epicentre of the first Industrial Revolution in Spain, which would have an impact on the incorporation of scientific advances in the restoration discipline. Moreover, the development of Catalonian nationalism, in reply to the centralism of Castilian Spain, would mark it with some of its own particularities. In this text the features of the gestation of two differentiated models of institutional restorers are presented.

Key words: Restorers, Madrid, Barcelona, institutionalisation, centralism, nationalism, professional specialisation. 


\section{Introducción}

La configuración de la figura del restaurador decimonónico, dentro del ámbito institucional español, estuvo sujeta a la interrelación de múltiples elementos sociohistóricos que fueron determinantes en el impulso y reconocimiento del perfil profesional del restaurador. En este caso, estudiamos lo sucedido en las ciudades de Madrid y Barcelona, a partir de fuentes documentales, para observar cómo incidieron dichos factores en contextos geográficos determinados.

Por lo que respecta a Madrid, cabe señalar que la decisión de Felipe II, en 1561, de trasladar la Corte a esta ciudad condicionó su posterior desarrollo y su notabilidad. La progresiva llegada de nobles, intelectuales y artistas atraídos por la Corte ${ }^{1}$ llevó a Madrid a suplantar el mecenazgo artístico que había disfrutado Sevilla en épocas precedentes. Además, en el siglo XIX, el desarrollo de los Estados Nacionales se articuló sobre la construcción de una identidad cultural propia sustentada en la recreación del pasado común. De hecho, los referentes identitarios sirvieron para legitimar los proyectos políticos; muestra de ello fue la creación de los grandes museos en las diferentes capitales europeas (Prats 1997; Santamarina 2005). En este siglo la ciudad del Manzanares, como capital del Estado, se convirtió en centro neurálgico, concentrando las principales instituciones encargadas de supervisar y gestionar la conservación y restauración nacional. En este sentido, el centralismo madrileño justificó el protagonismo de la Real Academia de Bellas Artes de San Fernando como principal órgano de control de las restauraciones y del patrimonio ${ }^{2}$. Hasta tal punto que esta institución impulsó las bases del perfil profesional del restaurador en el ámbito institucional español.

En cuanto a Barcelona, esta ciudad ha sido históricamente un centro político y cultural de enorme relevancia, entre otras razones, por su situación estratégica como puerta de Europa. Su posición privilegiada la convirtió muy pronto en uno de los principales puertos del Mediterráneo. En particular, desde el siglo XVIII se produjo un impulso espectacular del comercio y de la industria que revirtió, por ejemplo, en la financiación de proyectos urbanísticos -como fue el de las Ramblas- o en la creación de las primeras academias catalanas. En el siglo XIX acontecieron una serie de transformaciones socio-políticas que vinieron a consolidar la ciudad como un polo económico y cultural de primer orden. Por un lado, la división provincial (1833) convirtió a Barcelona en capital de provincia. Y, por otro, la proclamación de la libertad de industria (1836), permitió un incipiente desarrollo industrial catalán. Gracias a la Revolución Industrial desplegada se consolidó una potente burguesía que convirtió a la ciudad Condal en el primer centro industrial del país. Asimismo, se gestó un importante movimiento cultural e identitario conocido como la Renaixença. Desde mediados del XIX, el crecimiento económico y cultural de la capital catalana se vio reflejado en la reorganización y ampliación de la ciudad, con el plan del Eixample de Idelfonso Cerdá (1859). Reestructuración que quedó patente con la Exposición Universal de 1888, cuando Barcelona buscó y alcanzó la tan ansiada proyección internacional.

En síntesis, frente al modelo de centralismo madrileño asistiremos al desarrollo de un nacionalismo catalán; ambos nacionalismos impulsaron el patrimonio y su conservación de formas diferenciadas. Teniendo presente estos contextos, pasamos ahora a analizar los principales acontecimientos y figuras que determinaron las características de cada lugar.

\section{Situación de los restauradores madrileños del siglo XIX}

En la primera mitad del siglo XIX comenzó la progresiva desvinculación de la figura del restaurador respecto del artista, cuya máxima expresión llegó de la mano del restaurador José Bueno (1797-1849). Aunque tenía una formación artística, su intensa trayectoria profesional al servicio de las principales instituciones madrileñas que contaban con talleres de restauración le llevó a reivindicar una consideración distinta y autónoma para el restaurador.

Haciendo un rápido repaso a su trayectoria, sabemos que en 1815 comenzó a trabajar como ayudante temporal de Vicente López que, por aquel entonces, compaginaba su labor como primer Pintor de 
Cámara con la restauración de las obras del Palacio Real ${ }^{3}$. Inaugurado el Real Museo de Pintura y Escultura, en noviembre de $1819^{4}$, se formó en él un primer equipo de pintores-restauradores para acometer las intervenciones. Conforme la colección fue aumentando, se hizo preciso recurrir a nuevos ayudantes. De tal modo que en paralelo al crecimiento vertiginoso del museo, se produjo un incremento paulatino de la plantilla que conformaba este incipiente taller, con vistas a poder atender un mayor volumen de obras. Esto requirió unos patrones de organización cada vez más complejos, en base a las diferentes funciones y perfiles.

Desde entonces, Bueno inició una carrera ascendente alcanzando, en 1823, el cargo de ayudante del primer Pintor de Cámara por Real Orden. Como hecho significativo, en 1827 se creó en el Real Museo de Pintura y Escultura una Sala de Restauración de nueva planta ${ }^{5}$. En ella había dos inspectores como principales responsables: López y el también Pintor de Cámara Juan Antonio Rivera, encargados de la vigilancia, control y asesoramiento de las intervenciones. Dadas las buenas aptitudes de los ayudantes del taller, en 1828 se accedió al nombramiento real de José Bueno, Victoriano Gómez y Pedro Bueno como primer, segundo y tercer restaurador, con el sueldo anual de doce mil, once mil y ocho mil reales respectivamente ${ }^{6}$. Los datos acerca de los aumentos y diferencias salariales vienen a ser un claro indicador del reconocimiento del estatus laboral. Con este nombramiento, los tres pasaban a gozar de la consideración de ser criados de S.M. en la clase de la Real Cámara, con el consiguiente derecho a los auxilios de facultativos y medicinas, a disfrutar de los beneficios del Monte Pío de la Real Casa y a jurar sus plazas. Pero lo más importante es que este sistema anticipaba una estructura piramidal, en base a un orden claramente jerarquizado de puestos diversificados. En ella cobraba protagonismo un perfil profesional emergente, el del restaurador, que se veía distanciarse así de los pintores. Por tanto, éste fue el primer centro institucional español que impulsó una estructura laboral en el taller, planteando una ordenación y jerarquización por categorías.

En 1829 aconteció un episodio que determinaría el giro definitivo de la carrera de Bueno: la propuesta del rey Fernando VII de que éste marchase a Francia para aprender el procedimiento de trasposición de soportes pictóricos, que constituía una de las operaciones más arriesgadas de la época. El conocimiento que tenía Bueno de este procedimiento, y que aplicó con éxito en una pintura flamenca, le valió el nombramiento como Académico de Mérito por la Pintura de la Real Academia de Bellas Artes de San Fernando en 1829.7

Con todo, su mayor reconocimiento llegaría unos años más tarde, tras obtener por Real Orden, en 1831, el nombramiento como Restaurador de Cámara, "sin sueldo alguno, pero con el uso del uniforme" 8 distintivo que permitiera diferenciar a los Restauradores de los Pintores de Cámara". En el plano simbólico, el hecho de asignar un uniforme implicaba otorgar una identidad y, por tanto, un reconocimiento oficial y autónomo para este colectivo profesional. Por todo ello, deberíamos matizar la creencia general que atribuye al famoso restaurador italiano Giovanni Secco Suardo la primera reivindicación profesional de separar la figura del pintor y la del restaurador. A pesar de la modernidad del Manuale ragionato per la parte meccanica del Arte del restauratore dei dipinti (1866), en España, José Bueno ya había reivindicado, 35 años antes, esta diferente consideración profesional.

De forma casi coetánea, en 1831 se redactaron dos reglamentos en los cuales se concretaban las funciones y competencias propias de cada uno de los cargos existentes en la Sala de Restauración de Pinturas y Esculturas del Real Museo. Ambos reglamentos tenían en común una misma estructura, en base a una estricta clasificación por categorías y funciones. Además, planteaban una escala de profesionales, perfectamente organizada y regulada, para las restauraciones pictóricas y escultóricas.

El avance hacia un mayor grado de definición llevó a Bueno, incluso, a plantear la necesidad de crear una Escuela de Restauración en el Real Museo de Pintura y Escultura, tal y como plasmó en el Memorial para mejorar y asegurar los adelantos del ramo de la restauración, que presentó en 1833 a López y al duque de Híjar. En este documento solicitaba el nombramiento en propiedad para los restauradores temporeros y se ofrecía a instruir a algunos jóvenes pensionados bajo dirección como primer Restaurador de Cámara. Con todo, 
conviene destacar que esta Escuela, más que erigirse como un centro reglado de enseñanzas teóricoprácticas, se concibió más bien como un espacio para la formación de restauradores a partir de su aprendizaje diario, que debía ser guiado y controlado por los dirigentes de la sala. Pero más allá de estos avances en el plano docente, Bueno reconocía a los restauradores de plantilla y reivindicaba la promoción interna de los mismos, solicitando unos erarios y derechos acordes con su categoría laboral. En este sentido, planteaba incentivos a los alumnos que diesen muestras de sus progresos en la materia. En suma, esta propuesta se nos revela fundamental por su modernidad. Aunque no llegó a tener el alcance que Bueno hubiese deseado debido a la precariedad de las finanzas públicas, que terminarían por diluir este proyecto (Ruiz Gómez 2007), tras ella se entrevé el deseo de formar unos perfiles profesionales claros y definidos en el ámbito de la restauración, que conduzcan a la concepción de una estructura laboral más compleja y coherente con las necesidades del oficio.

En 1833, y en paralelo a sus servicios como restaurador en el Museo Real, Bueno decidió solicitar su entrada como restaurador en la Real Academia de San Fernando "sin sueldo alguno"10, tan sólo por el privilegio que suponía trabajar en el seno de la Academia (que no dejaba de ser una institución regia), y comprometiéndose a formar a dos jóvenes para desarrollar las labores de restauración y a un tercero como forrador. Es significativo que para los dos primeros estableciera como condición un conocimiento previo de la pintura, sin hacer esta distinción en el último caso. Al respecto conviene precisar que, en este tiempo, el forrador se encargaba de ejecutar determinados procedimientos mecánicos (principalmente, los reentelados), mientras que al restaurador se le confiaba, sobre todo, el retoque pictórico de las lagunas. Con ello se abría una brecha entre el restaurador, de indiscutible habilidad con el pincel, y los demás operarios del taller (forrador, engatillador de tablas, moledor de colores, etc.), de categoría y remuneración inferior al primero. Esto nos confirma la diferente consideración social existente en la disciplina. Finalmente, en 1835 se resolvió designar a Bueno restaurador de los cuadros de la Real Academia de Bellas Artes de San Fernando.

En 1847 llegó el nombramiento de José Bueno como Jefe de Restauración de Pinturas del Real Museo; plaza que juró fielmente a la reina Isabel $\mathrm{II}^{11}$. Falleció dos años después, tras más de treinta años de servicio, erigiéndose en una figura clave de la restauración de pinturas en el ámbito institucional de la Corte madrileña decimonónica.

En la segunda mitad del siglo XIX, la hegemonía del Real Museo de Pintura y Escultura se amplió al Museo Nacional de Pinturas y Esculturas (más conocido como Museo Nacional de la Trinidad por su emplazamiento en el desamortizado convento trinitario de la calle Atocha de Madrid). Este nuevo museo se inauguró en 1838 y en él se creó otro taller de restauración para atender, de forma específica, las obras requisadas en diferentes conventos castellanos tras la Ley de Desamortización impulsada por el ministro de Hacienda, Juan Álvarez Mendizábal (1835-1837). La proximidad y relevancia de ambos talleres hizo constante la sucesión de restauradores en ellos, lo que llevó a un sinfín de encargos, nombramientos y variadas categorías profesionales hasta que, en 1872, el Gobierno de la Primera República decidió fusionar los dos museos.

Desde 1853, gracias al Libro de apuntaciones de la Sala de Restauración de Pinturas del Real Museo de S.M. podemos conocer el volumen de las restauraciones pictóricas realizadas en este taller -diferenciando los procesos de forración y los de restauración-. En definitiva, constituyó un inventario de las restauraciones efectuadas, en el que los perfiles profesionales quedaban clasificados en base a sus funciones y a su distinta consideración. En este sentido, el hecho de que se especificase el nombre del restaurador pero no el del forrador viene a ser un claro indicador de esta jerarquización.

Por lo que respecta al Museo Nacional de la Trinidad, las operaciones de restauración efectuadas en este taller fueron análogas a las del Museo Real. Pedro Kuntz, como director de restauración en el taller de la Trinidad, redactaba a principios de cada mes una breve relación de las tareas ejecutadas, indicando el nombre del restaurador, una reseña de la obra que permitiera su localización y una somera descripción de los trabajos efectuados ${ }^{12}$ [Figura 1]. Gracias a esto tenemos constancia de los procesos de intervención más frecuentes, destacando las forraciones a la gacha y los engatillados. Otro de los procedimientos más 
repetidos y controvertidos, al ver después sus consecuencias, consistió en rebajar el grosor de las tablas originales para paliar los alabeos de la madera (Ruiz Gómez 2001). Aunque al principio estos trabajos los ejecutaron los mismos forradores, conforme avanza el siglo fueron encomendados a carpinteros especializados. Esto repercutió en la constitución de equipos profesionales cada vez más sólidos, con un reparto de competencias en función de los diferentes perfiles.

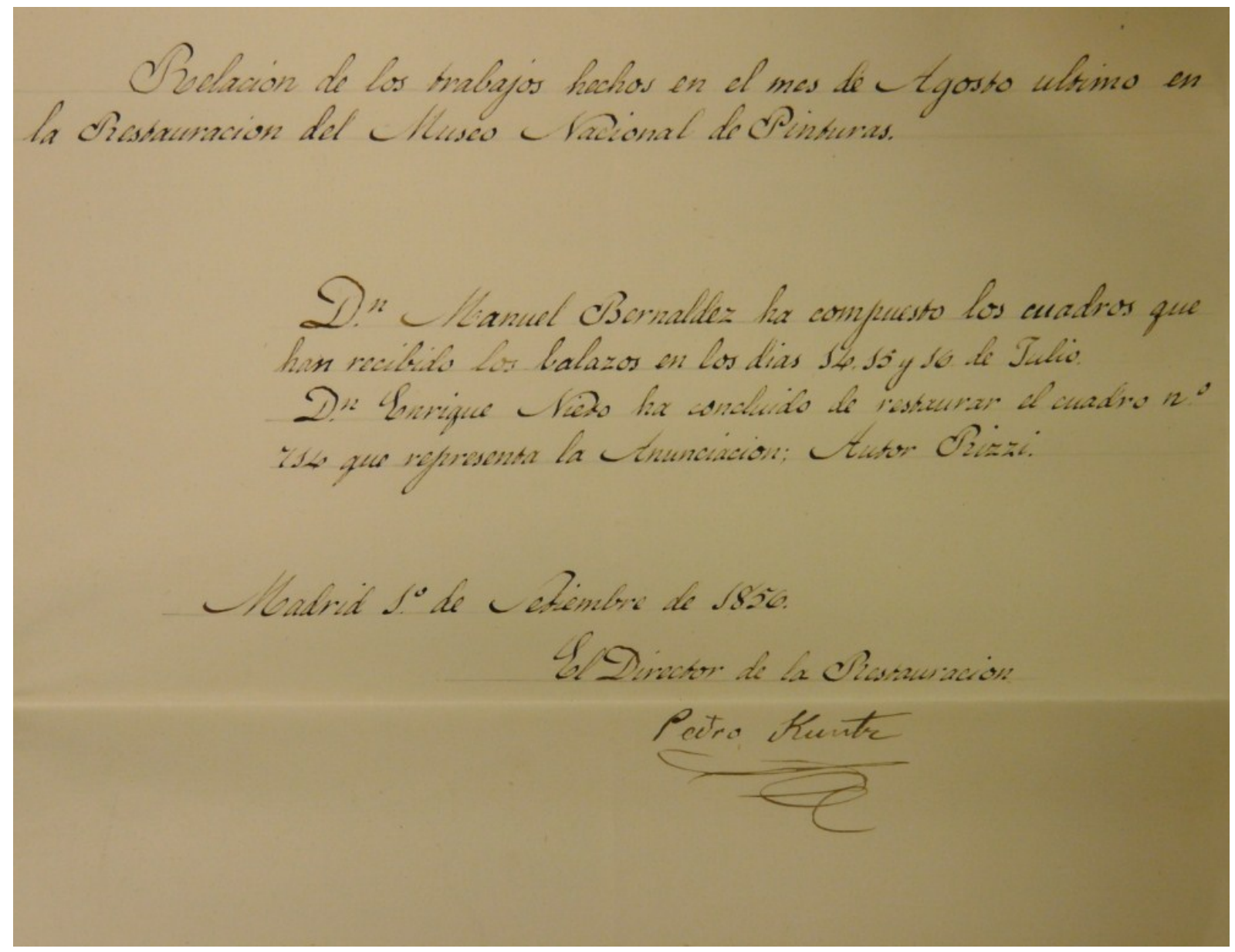

Figura 1. Relación de las restauraciones registradas en el Libro de apuntaciones de la Sala de Restauracion de pinturas del Real Museo de S.M., con fecha de 1 de septiembre de 1856. Archivo del Museo Nacional del Prado.

Con todo, la segunda mitad del siglo XIX estuvo marcada por frecuentes problemas económicos que obligaron a continuas cesiones y reestructuraciones entre la plantilla en ambas instituciones. De hecho, en 1866 se aprobó un Real Decreto por el que se reducía la plantilla del Museo Real, siendo el departamento más afectado el de restauración. Esto implicó numerosos despidos y la suspensión temporal de las forraciones. Dichas medidas se hicieron también extensivas al taller del Museo Nacional, donde ese mismo año se ordenó la supresión de la plaza del forrador de cuadros Antonino de Manuel, que éste había ganado por oposición.

En 1868, al iniciarse la denominada Revolución Gloriosa, el Museo Real quedó cerrado cautelarmente (Alcolea 2002). Tras su nueva apertura, la institución pasó a denominarse Museo Nacional del Prado y a depender del Estado. Este cambio, más acorde con las ideas liberales que impulsaron la revolución, respondía a la transición del patrimonio privado al público donde cobraba relevancia, bajo la idea de la nación, el carácter colectivo y, en consecuencia, social de los bienes susceptibles de ser conservados. En consonancia con ese gran proyecto de 'museo nacional' se formuló, incluso, la idea de fundir en uno los 
dos museos existentes: el Prado y la Trinidad. Así lo plasmó, en octubre de 1868, Vicente Poleró en sus Breves observaciones sobre la utilidad y conveniencia de reunir en uno solo los dos museos de pintura de Madrid y sobre el verdadero estado de conservacion de los cuadros que constituyen el Museo del Prado, aunque esta anexión no se produjo hasta 1872, quedando la colección bajo custodia del director del Prado. Esto supuso el cese de muchos de los restauradores de las dos instituciones, principalmente, de la Trinidad.

Con todo, frente a dichas dificultades, en las últimas décadas del XIX podemos resaltar varios hechos de singular relevancia en materia de restauración, en la medida que reclamaron una mayor especialización profesional y una división del trabajo. Uno de los ejemplos más claros lo hallamos en 1867, cuando Gregorio García solicitó que se replantease su condición profesional al pasar de carpintero a carpinteroengatillador de tablas. Incluso, justificaba este aumento de sueldo reconociendo la importancia de un trabajo específico y delicado que, en su opinión, el director del museo debía proteger ${ }^{13}$. Por tanto, sus palabras anticipan una defensa de la situación profesional del restaurador. De forma análoga, en 1882 Antonino De Manuel fue nombrado 'Restaurador especialista para pasar tablas á lienzos en el Museo Nacional de Pintura y Escultura' [Figura 2]. De tal modo, en estos años se diferenció entre el trabajo del forrador, José Muñiz, y el del restaurador especializado en las trasposiciones de soportes ${ }^{14}$.

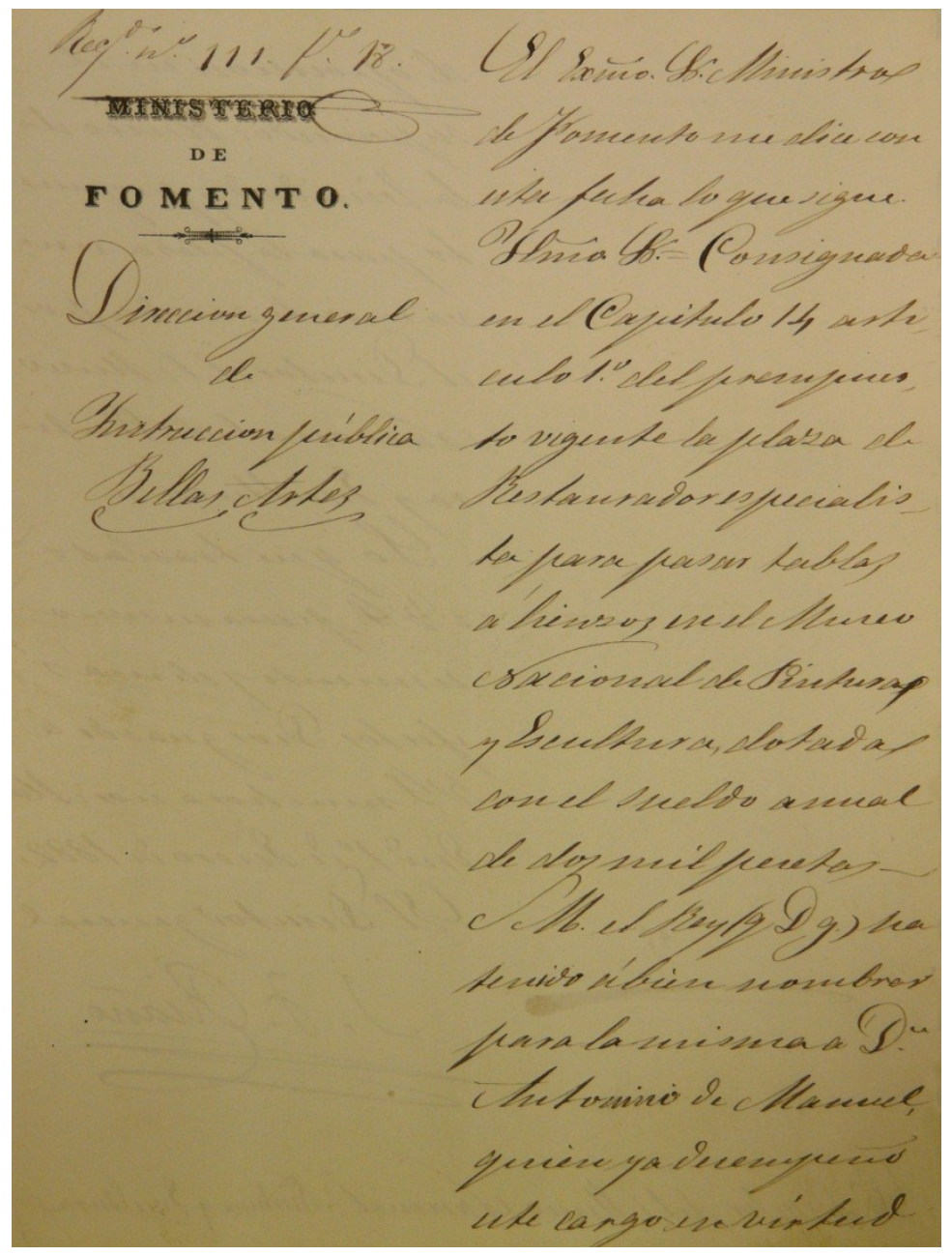

Figura 2. Nombramiento de Antonino de Manuel para la plaza 'Restaurador especialista para pasar tablas á lienzos en el Museo Nacional de Pintura y Escultura' (1882). Archivo del Museo Nacional del Prado. 
Este mismo deseo de especialización estuvo, también, en la base de los viajes de formación que algunos restauradores emprendieron al extranjero, con objeto de incorporar los avances técnicos de otros museos europeos. Es el caso de José Rivero, restaurador cuarto del Museo Nacional de la Trinidad, que en 1864 fue comisionado seis meses para completar su formación en otros talleres nacionales e internacionales ${ }^{15}$. Este hecho constata la importancia de alcanzar una formación de calidad que se adecuase a los sistemas y procedimientos que, por estas fechas, se llevaban a cabo en otros talleres de restauración. Por tanto, esta iniciativa constituye un referente en cuanto al intercambio de conocimientos entre profesionales que redundará en la mejora y el avance de la disciplina.

Asimismo, la proliferación de nombramientos y la diversificación de funciones contribuyeron a un mayor grado de especialización, y alentaron la necesidad de convocar, desde mediados del XIX, las primeras plazas por oposición. A través del programa de este tipo de concursos se evidencia la misma evolución del oficio, pues de la predominancia de pruebas artísticas se va hacia otras que priorizan los conocimientos teórico-prácticos en restauración. En ellos queda de manifiesto la relevancia que adquirió la Real Academia de Bellas Artes de San Fernando, a la que correspondía la revisión y el dictamen de las intervenciones, el establecimiento de los programas de oposición a la plaza de restaurador, así como el Tribunal que debía evaluar estos ejercicios. De ahí que la presencia de esta institución determinó, sin duda, la actividad del restaurador en estos años.

Por último, no podemos concluir este apartado sin mencionar que todos los avances anteriores fueron coetáneos a las publicaciones de tratados e informes de restauración. En ellos se advierte una notable diferencia respecto a la tratadística precedente, ya que no sólo recogen fórmulas y recetas, sino que anticipan algunas consideraciones sobre los procesos de restauración. Quizás el caso más conocido sea el del restaurador, académico y uno de los primeros teóricos españoles de la conservación y restauración Vicente Poleró y Toledo (1824-1899), que en 1853 sacó a la luz su famoso tratado Arte de la Restauración, observaciones relativas a la restauración de cuadros. En él trató de sistematizar los principales procesos de restauración aplicados en España, de acuerdo con un planteamiento más moderno, distanciándose de los anteriores tratados de recopilación de fórmulas y focalizando la relevancia de este escrito en los criterios de actuación. Así, destacó la necesidad de fomentar la conservación y de limitar o restringir las intervenciones -sobre todo, en los procesos de limpieza, estucado y reintegración-, a la vez que denunció los casos de restauraciones anteriores inadecuadas que habían producido en las obras nuevas patologías. En línea con lo anterior, Mariano de la Roca y Delgado, en 1880, publicó el tratado Compilación de todas las prácticas de la pintura, desde los antiguos griegos hasta nuestros días, que completó con un apartado dedicado a la limpieza, forración y restauración de los cuadros. En suma, con ellos no sólo se impulsa la crítica de la restauración, sino que, a su vez, esta producción teórico-crítica será indicativa de la aproximación del método científico.

\section{Situación de los restauradores catalanes del siglo XIX}

Si Bueno personifica en Madrid la transformación en la consideración del restaurador, en Barcelona destaca con nombre propio la figura de Josep Arrau i Barba por ser pionero en la redacción de ensayos de restauración durante la primera mitad del siglo XIX [Figura 3]. Dejando de lado su enorme talla intelectual ${ }^{16}$, el aspecto más interesante para nuestro estudio es la redacción de varios ensayos sobre restauración sin parangón en España. Ya hemos visto que los tratadistas madrileños son de la segunda mitad del siglo, por lo que los escritos de Arrau i Barba, aún siendo menos conocidos, constituyen un auténtico precedente para comprender el desarrollo y consolidación de la restauración.

No podemos extendernos en su obra, pero queremos al menos destacar algunas de sus aportaciones. En este sentido, si atendemos a su discurso de ingreso en la Academia de Ciencias Naturales, Sobre el modo de limpiar y restaurar las pinturas antiguas (1834), veremos reflexiones que son verdaderamente transgresoras para su tiempo. El texto aborda desde consideraciones técnicas hasta la denuncia de los abusos cometidos por personas inexpertas. Con todo, es interesante anotar cómo trata las principales causas de deterioro en la pintura. Así, en su escrito, reconoce la especificidad e individualidad de cada factura (rasgo moderno, 
pues con él invalida el uso de recetas universales) y los distintos factores (medioambientales y lumínicos) que influyen en la conservación de la obra. De forma que reclamó la aplicación de una metodología concreta según cada caso e hizo un llamamiento en favor de los nuevos métodos que permitieran reparar los errores cometidos en el pasado (Xarrié 2002). Al mismo tiempo, reafirmó la necesidad de salir del hermetismo imperante en los talleres precedentes y hacer público el conocimiento de los avances científicos. De este modo, anticipó una consideración abierta y multidisciplinar de la restauración que constituirá una de sus principales señas de identidad en adelante. En suma, su postura es acorde con su contexto, determinada por la convivencia entre el incipiente desarrollo científico y el arte de pintar y restaurar, límite aún muy difuso.

Pese a la impronta de Arrau i Barba en las primeras décadas del XIX, es posible rastrear el desarrollo del restaurador catalán de la segunda mitad del siglo a través de las instituciones. En ese periodo la Galería de Pinturas de la Academia de Sant Jordi fue ampliándose con nuevas adquisiciones y fue adquiriendo, progresivamente, carácter de museo. No obstante, la falta de recursos había llevado a que la colección estuviera en un estado deplorable, y el hecho de que fuesen las únicas obras de apoyo a la docencia, justificaba su intervención inmediata. A esto se añadía una infraestructura deficiente. Por ello, en 1858, el profesor Jaime Batlle propuso llevar a cabo una reforma de la Galería de Pinturas y aprovechar para proceder a la reordenación, consolidación, limpieza y enmarcación de numerosas obras. Fue entonces cuando se contempló la adecuación de un local específico para acometer las restauraciones. La asignación de este espacio es un claro indicador de la importancia que adquiere la restauración ${ }^{17}$.

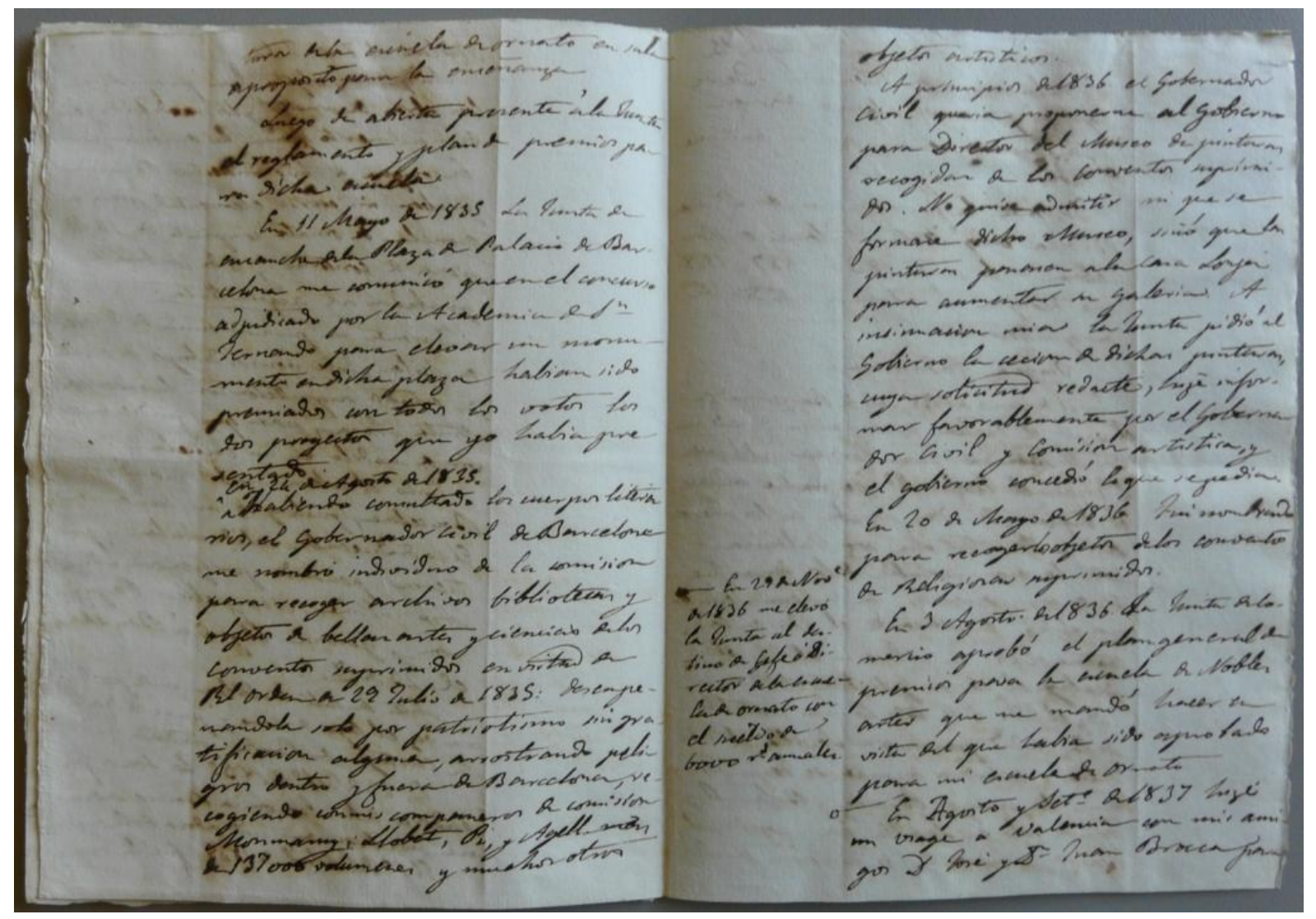

Figura 3. Fragmento del documento de Josep Arrau i Barba titulado 'Apuntes: para mi biografía', donde recoge su participación en la Comisión de Incautación entre 1835 y 1836. Biblioteca del Museo Nacional de Arte de Cataluña. 
Pese a todo, no fue hasta principios de los años sesenta cuando, por fin, se destinó una significativa partida presupuestaria para la restauración, que desde 1860 estuvo en manos del restaurador Miguel Aragón. En las cuentas archivadas en la Academia se recogieron, aunque muy escuetas, las operaciones ejecutadas, el orden de los trabajos y el salario del restaurador [Figura 4] ${ }^{18}$. De esta fuente documental podemos extraer que frente a la diversificación de perfiles de Madrid, en Barcelona todas las tareas convergían en la misma persona. Tal vez, el motivo se deba al menor número de fondos que conformaba la colección catalana. Por una parte, este factor estaría determinado por el centralismo político, que condicionó que los bienes que formaban el Real Museo de Pintura y Escultura de Madrid procedieran, en gran parte, de las colecciones reales, mientras que en el caso de Barcelona su origen principal estaba en la recuperación de obras desamortizadas y en la práctica del coleccionismo privado. Por otra parte, las diferencias en cuanto al volumen de la colección se explican atendiendo a la antigüedad de San Fernando respecto a la Academia barcelonesa. En este sentido, las instituciones madrileñas son bastante anteriores en el tiempo a las restantes de España.

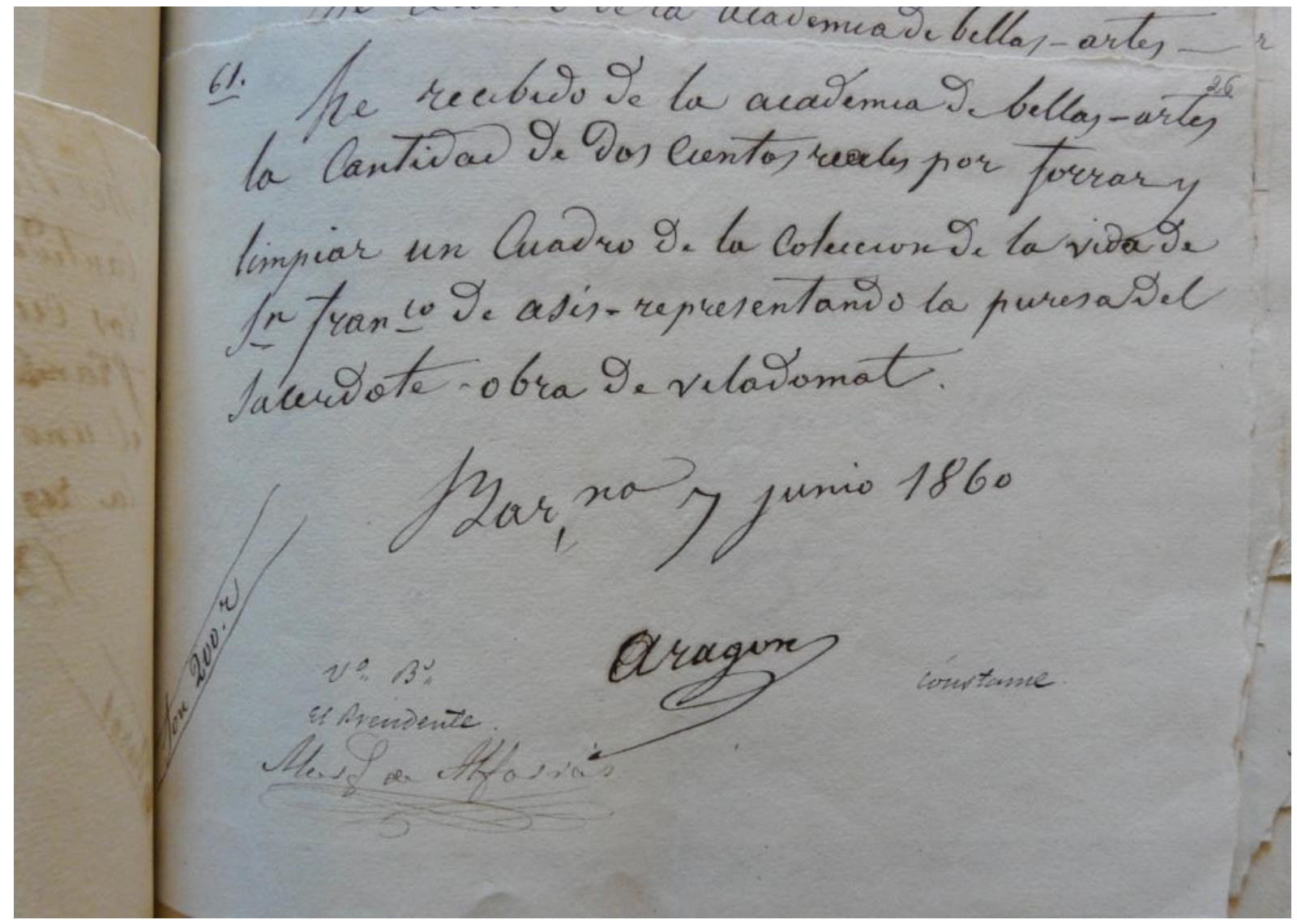

Figura 4. Ejemplo de una de las cuentas archivadas en la Academia que recoge el trabajo realizado por Miguel Aragón y el sueldo que percibió por ello (1860). Archivo de la Real Academia Catalana de Bellas Artes de Sant Jordi.

En relación con lo anterior, la Exposición Nacional de Objetos de Arte organizada por la Academia en 1866, permitió la adquisición de numerosas obras expuestas y otras procedentes del Museo del Prado en calidad de depósito. En Madrid se nombró una Comisión encargada de seleccionar y acompañar durante su traslado los cuadros del Museo Nacional. Al llegar a Barcelona, la Comisión vio conveniente la creación de una plaza de restaurador para atender, de forma específica, las obras existentes en la Academia catalana ${ }^{19}$. El marqués de Alfarrás, director de esta institución, se lamentó de no poder atender esta 
recomendación, en base al bajo presupuesto, al corto número de cuadros que poseía (de los cuales ya se habían restaurado los que debían), y a la dificultad de poder adquirir en lo sucesivo otras obras de mérito. Con ello constatamos que, pese a la importancia cada vez mayor de esta colección, sus fondos estaban muy lejos de justificar la creación de una plaza fija de restaurador. No obstante, nuevos ingresos y adquisiciones desde finales de los 60 alentaron el deseo de perfilar un auténtico Museo Provincial de Bellas Artes y desde Madrid se enviaron nuevos depósitos. Esto, a su vez, motivó la redacción de un catálogo que incluía, entre otra información complementaria, las restauraciones realizadas en esos años. Este hecho supone un salto cualitativo, en la medida que implica una mayor sistematización de la disciplina.

Estos esfuerzos se vieron coartados por la Orden Circular de 9 de julio de 1880, que exigía la suspensión de las restauraciones en todos los museos provinciales. El objetivo de esta orden era garantizar el éxito de las operaciones de restauración en las obras más relevantes que, en lo sucesivo, debían ser autorizadas por la Real Academia de San Fernando. Como consecuencia de la Orden, se paralizaron las tareas de restauración, lo que justificaría la falta de documentación en esta materia hasta finales de los ochenta. El detonante que propició, de nuevo, el impulso de la restauración debió ser la Exposición Universal celebrada en Barcelona en 1888. La intensa labor de mejora que, con motivo de este certamen, se llevó a cabo en todos los ámbitos de la ciudad nos permite presuponer que también influyó en el marco de la restauración de bienes muebles. A la luz de estas circunstancias, no es extraño que fuera en 1888 cuando se autorizase la restauración de unos ochenta cuadros que se estaban deteriorando, de forma alarmante, en los desvanes del museo. Esta segunda oleada de restauraciones la llevó a cabo el artista-restaurador Mariano Bellver ${ }^{20}$, que fue el sucesor de Miguel Aragón en el ámbito de intervenciones pictóricas desde esta década.

Para finalizar, es importante reseñar que en el último tercio del XIX Barcelona vivió un enorme impulso museístico. Esto trajo como resultado la presencia de nuevos restauradores institucionales con perfiles especializados. Por poner un ejemplo, en el Museo de Reproducciones Artísticas encontramos a José Casanova Mones para restaurar los objetos metálicos, cerámicos y vítreos y a Casimiro Lucheri, para reparaciones escultóricas.

\section{Conclusiones}

El desarrollo de la restauración en España no puede ser entendida al margen, al menos, de dos procesos. Por una parte, la aparición del concepto de patrimonio, que estuvo impulsada en gran medida por el desarrollo en Europa de los Estados Nacionales. Y, por otra, el desarrollo científico (promovido por la Revolución Industrial), que dio como resultado la incorporación de nuevos procedimientos y materiales.

Teniendo presente ambos procesos, se entenderá que para comprender bien la especificidad del restaurador en cada una de las ciudades estudiadas es necesario atender a su contexto. De este modo, el protagonismo indiscutible de Madrid debe ser interpretado como centro político y cultural. Esto repercutió en que el despliegue abrumador de restauradores en Madrid diste tanto del crecimiento profesional en Barcelona. La razón radica en que las instituciones barcelonesas son mucho más recientes que las madrileñas, lo que condicionó el menor volumen de sus fondos y se reflejó en una demanda menor de restauradores. El protagonismo de los talleres de restauración madrileños resulta incuestionable dada su temprana configuración, su intensa actividad y el volumen de restauradores que trabajan en plantilla con unos patrones de organización cada vez más complejos.

En líneas generales podemos decir que en el XIX el avance del perfil del restaurador se produjo de un modo lento e irregular y, a finales de siglo, adolecía todavía del grado de definición profesional que se merece. No obstante, los grandes cambios que hemos analizado sientan la base de una concepción moderna de la restauración y permitirán explicar el despliegue de la disciplina en el siglo XX. Podemos decir, en consecuencia, que la situación del restaurador decimonónico presenta enormes contrastes. Por una parte, se reconoce la especificidad de su cometido, lo que derivó en perfiles cada vez más técnicos, en la convocatoria de los primeros concursos por oposición y en la necesidad latente de una formación 
reglamentada; de ahí que, para asumir la restauración de obras más emblemáticas, se recurriera al profesional más cualificado. Pero, por otra parte, su trabajo aún estaba definido por la no remuneración y por la irregularidad de su situación que se vio compensada por el prestigio social que se derivaba de su cometido. Además, dicha labor estuvo siempre expuesta al dictamen de personas de mérito, aquellas que se erigieron como custodias de la conservación.

\section{Notas}

[1] Uno de los ejemplos que mejor reflejan esta situación lo encontramos en el artista hispalense Diego Velázquez, que realizó su primer viaje a la Corte madrileña en 1621, estableciéndose en ella, dos años después, como Pintor de Cámara del rey Felipe IV.

[2] Al papel de la Real Academia de Bellas Artes de San Fernando cabría sumar el de las Comisiones Provinciales de Monumentos Históricos y Artísticos.

[3] Madrid, Archivo General de Palacio, Personal, caja 16694, exp.1.

[4] Junto a él encontramos a su compañero Victoriano Gómez, hijo del Pintor-Restaurador de Cámara del siglo XVIII Jacinto Gómez.

[5] Madrid, Archivo del Museo Nacional del Prado, Restauraciones, caja 77, leg. 23.01, exp.2.

[6] Madrid, Archivo del Museo Nacional del Prado, Restauraciones, caja 77, leg. 23.01, exp.3.

[7] Madrid, Archivo General de Palacio, Personal, caja 16694, exp.1.

[8] Íbidem.

[9] Para el diseño y bordado del uniforme de restaurador sabemos que se presentó un dibujo, encontrado en el Archivo General del Palacio Real.

[10] Madrid, Archivo de la Real Academia de Bellas Artes de San Fernando, signatura 1-45-6.

[11] Madrid, Archivo General de Palacio, Personal, caja 16694, exp.1.

[12] Madrid, Archivo del Museo Nacional del Prado, Restauraciones, caja 77, leg. 23.01, exp.16-25.

[13] Madrid, Archivo del Museo Nacional del Prado, Restauraciones, caja 77, leg. 35.03 A-82, exp.4.

[14] Íbidem.

[15] En la documentación de archivo consultada no se alude de forma explícita a los talleres que visitó ni a los procedimientos en los que se especializó. Tan sólo se especifica que la reina resolvió comisionarlo para completar su formación, durante los tres primeros meses, en los talleres de restauración extranjeros, y durante los tres meses siguientes en los talleres de las provincias españolas de Castilla y Andalucía. Madrid, Archivo del Museo Nacional del Prado, Restauraciones, caja 77, leg. 35.03 A-82, exp.20.

[16] Josep Arrau i Barba (1802-1872) fue un destacado pintor académico y uno de los mayores intelectuales de su tiempo. Pensionado por la Junta de Comercio, en 1819 inició una fructífera trayectoria artística en la Casa Lonja de Barcelona. Prueba de su reconocimiento artístico fueron los numerosos premios recibidos y el encargo de retratar, en 1832, al rey Fernando VII y a la reina María Cristina. Todo ello motivó su nombramiento como Académico de Mérito de la Real Academia de Bellas Artes de San Fernando en 1833. En paralelo desarrolló una ambiciosa carrera científica y humanista (estudiando, entre otras disciplinas, Notarías, Anatomía, Química, Botánica o Mineralogía). Fue, además, uno de los principales integrantes de la Comisión de Incautación encargada de seleccionar y salvaguardar el patrimonio cultural catalán durante las vicisitudes políticas de 1835 , y de dirigir en 1836 el proyecto museístico para albergar los fondos de los 
conventos suprimidos. Para una aproximación a su figura se puede consultar en la Biblioteca del Museo Nacional de Arte de Cataluña numerosos documentos manuscritos que el propio Arrau i Barba redactó en primera persona, a modo de borrador, con el título Apuntes: para mi biografía.

[17] Barcelona, Archivo de la Real Academia Catalana de Bellas Artes de Sant Jordi, caja 64, exp.22.

[18] Barcelona, Archivo de la Real Academia Catalana de Bellas Artes de Sant Jordi, Libro de Actas de las Juntas de Gobierno 1861-1880.

[19] Barcelona, Archivo de la Real Academia Catalana de Bellas Artes de Sant Jordi, caja: Museo, carpeta: Restauraciones.

[20] Barcelona, Archivo de la Real Academia Catalana de Bellas Artes de Sant Jordi, Cuentas correspondientes al año 1887-1888.

\section{Bibliografía}

ALCOLEA BLANCH, S. (2002). Museo del Prado. Barcelona: Polígrafa.

BOHIGAS TARRAGÓ, P. (1947). "Resumen histórico de los Museos de Arte de Barcelona. I", Anales y Boletín de los Museos de Arte de Barcelona, 5: 129-168.

CARRETERO MARCO, C. (2002). "La restauración de pintura en el Museo del Prado en el siglo XIX. Vicente Poleró y el Real Sitio de San Lorenzo de El Escorial”. En I Congreso del Patrimonio: evolución y nuevas perspectivas, CD-ROM, Valencia: Grupo Español del IIC.

CARretero Marco, C. (2005). "Restauración en el siglo XIX. Materiales, técnicas y criterios". En II Congreso del GE-IIC. Investigación en Conservación y Restauración, Barcelona: Universidad de Barcelona, 169-179.

DÍAZ MARTOS, A. (1972). “Aportaciones a la Historia de la Restauración en España. Reimpresión de los tratados de Poleró y de la Roca con los informes del Restaurador Gato de Lema', Informes y trabajos del Instituto de Conservación y Restauración de Obras de Arte, 12: 101-135.

FONTBONA, F. (1986). "El Museu de la Reial Acadèmia Catalana de Belles Arts de Sant Jordi (1775), primer museu d'art de Catalunya', Butlletí de la Reial Acadèmia Catalana de Belles Arts de Sant Jordi, VII-VIII: 167-186.

MACARRÓN MIGUEL, A. M. (2002). Historia de la conservación y la restauración: desde la antigüedad hasta el siglo XX. Madrid: Tecnos.

MIRAMBELL ABANCÓ, M. (2002). "Del restaurador d'obres d'art al conservador-restaurador de béns culturals. Breu recorregut per la història de la professió", Unicum, 1: 6-11.

POLERÓ Y TOLEDO, V. (1855). El arte de la Restauración. Observaciones relativas a la Restauración de cuadros. Madrid: Imprenta M. A. Gil.

POLERÓ Y TOLEDO, V. (1868). Breves observaciones sobre la utilidad de reunir en uno solo, los dos museos de pintura de Madrid, y sobre el verdadero estado de conservación de los cuadros que constituyen el Museo del Prado. Madrid: Establecimiento Tipográfico de Eduardo Cuesta.

PRATS, Ll. (1997). Antropología y Patrimonio. Barcelona: Ariel.

RUIZ DE LACANAL RUIZ-MATEOS, M. D. (1994). Conservadores y Restauradores en la Historia de la Conservación y Restauración de Bienes Culturales: estudio del perfil y la formación. Madrid: Gráficas Olimpia.

RUIZ GÓMEZ, L. (2006). “Restauración en el Museo del Prado”, Enciclopedia del Museo del Prado, 5: 1836-1843.

RUIZ GÓMEZ, L. (2007). “Algunas notas sobre el restaurador José Bueno y la creación de la Escuela de Restauración del Museo del Prado”. En In Sapientia Libertas. Escritos en homenaje al profesor Alfonso E. Pérez Sánchez. Madrid-Sevilla: Museo Nacional del Prado y Fundación Focus-Abengoa, 670-676.

SACS, J. (1929). “Josep Arrau i Barba”, D’ ací I d'allà: 98-108. 
SANTAMARINA, B. (2005). "Una aproximación al patrimonio cultural”. En G. Hernández, B. Santamarina, A. Moncusí y M. Albert: La memoria construida. Patrimonio cultural y modernidad. Valencia: Tirant Lo Blanch, 21-51.

XARriÉ ROVIRA, J.M. (1975). “Josep Arrau, restaurador”, A Estudios Pro Arte, 3: 62-69.

XARRIÉ ROVIRA, J.M. (2002). Restauració d'obres d'art a Catalunya. Barcelona: Publicacions del'Abadia de Montserrat.

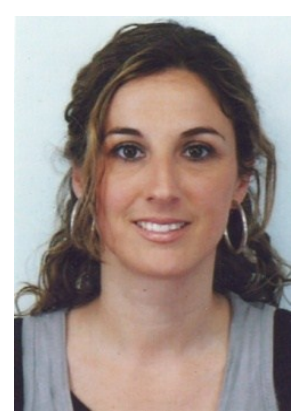

\section{$\mathbf{M}^{\mathrm{a}}$ Teresa Vicente Rabanaque}

Universidad Politécnica de Valencia, Camino de Vera s/n, 46022, Valencia. tevira@dmea.upv.es

María Teresa Vicente Rabanaque, es licenciada en Bellas Artes por la Universidad Politécnica de Valencia y en Historia del Arte por la Universidad de Valencia. Doctorado Europeo en Conservación y Restauración de Bienes Culturales por la Universidad Politécnica de Valencia, habiendo recibido el Premio Extraordinario de Tesis Doctorales convocado por esta universidad en 2011. Ha colaborado en diferentes publicaciones y proyectos de investigación sobre Historia de la Restauración y Patrimonio Cultural. En la actualidad, tiene un contrato como Técnico Superior con Grado de Doctor en el Instituto de Restauración del Patrimonio (UPV).

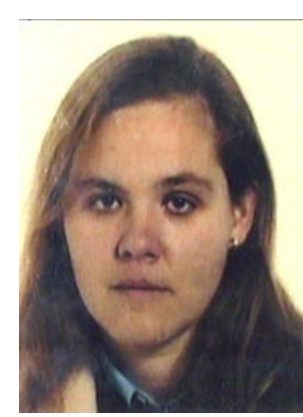

\section{Beatriz Santamarina Campos}

Av. Tarongers, 4 B. Departamento de Sociología y Antropología Social. 46021 Valencia Beatriz.Santamarina@uv.es

Beatriz Santamarina Campos, es licenciada en Ciencias Políticas y Sociología y en Geografía e Historia, y Doctora en Sociología por la Universidad Complutense de Madrid. Profesora Titular de Antropología Social, en la actualidad desarrolla su actividad docente e investigadora en el departamento de Sociología y Antropología Social de la Universidad de Valencia. Sus líneas de investigación actual giran en torno a procesos de patrimonialización cultural y natural. En este sentido, en este momento participa en varias investigaciones sobre patrimonialización y cuenta con diversas publicaciones sobre estos temas. Por último, ha impartido en Másteres y Doctorado de la UV y UPV docencia sobre patrimonio.

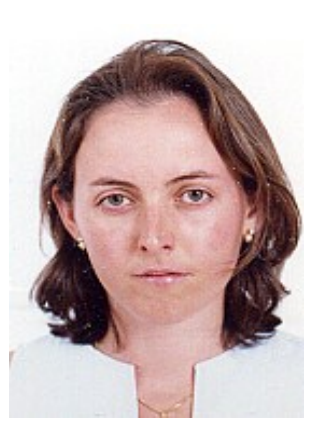

Virginia Santamarina Campos

Camino de Vera s/n, Departamento de Conservación y Restauración de Bienes Culturales (4 ${ }^{a}$ planta). 46022 Valencia.

virsanca@crbc.upv.es

Virginia Santamarina Campos, Profesora Titular de la Universidad Politécnica de Valencia, imparte docencia en grado y posgrado en el Departamento de Conservación y Restauración de Bienes Culturales de la UPV. Es integrante de los grupos I+D+I "Taller de Análisis e Intervención en Pintura Mural” y "Gestión sostenible del patrimonio cultural-natural" del Instituto de Restauración del Patrimonio de la UPV, y actualmente es coordinador del MicroClúster de Investigación (MCI) en VLC/CAMPUS "Globalización, terciarización, turismo y patrimonio: hacia una gestión sostenible del pasado como futuro".

Artículo recibido el 13/03/2011

Artículo aceptado el 13/06/2011 